\title{
TRES TÍTULOS DE ALFONSO DE CARTAGENA EN LA BIBLIOTECA CASANATENSE DE ROMA (MS. 389)*
}

\author{
Georgina Olivetto \\ Universidad de Buenos Aires - CONICET \\ golivetto@conicet.gov.ar
}

Más allá de los ciclos de traducciones de Cicerón y Séneca, cuya transmisión manuscrita es inusualmente homogénea ${ }^{1}$, la obra de Alfonso de Cartagena no suele encontrarse en códices de autor único. Los tres títulos que nos ocupan, la Qüestión sobre el fecho de la cavallería, el Doctrinal de los cavalleros y la versión castellana de la Propositio super altercatione prceminentia sedium, pueden localizarse alternativamente en misceláneos heterogéneos como el Ms. BNE 4236, que recoge tanto El conde Lucanor como la Proposición y la Qüestión ${ }^{2}$, o en misceláneos de homogeneidad marcada por el propio círculo de los Santa María, tal el caso de Esc. h-II-22, que junto a los mencionados textos, la Apología sobre el salmo «Judica me Deus» y la Glosa al tractado de san Juan Crisóstomo de Cartagena conserva, entre otros, las Siete edades del mundo de don Pablo y la Suma de las crónicas de España ${ }^{3}$.

\footnotetext{
${ }^{*}$ Este trabajo se inscribe en el marco del proyecto de investigación Alfonso de Cartagena. Obras Completas FFI 2014-55902-P y FFI 2017-84858-P (MINECO y MICINN, Gobierno de España).

${ }^{1}$ Como puede comprobarse en María Morrás, «Alfonso de Cartagena», en Diccionario Filológico de Literatura Medieval Española. Textos y transmisión, ed. de Carlos Alvar y José Manuel Lucía Megías, Madrid, Castalia, 2002, pp. 93-127.

${ }^{2}$ Descrito en el Inventario General de Manuscritos de la Biblioteca Nacional, X (3027-5699), Madrid, Ministerio de Cultura-Dirección General del Libro y Bibliotecas, 1984, pp. 306-307, y en Philobiblon BETA, manid 1675 (Charles B. Faulhaber, Ángel Gómez Moreno et al., BETA: Bibliografia Española de Textos Antiguos, 2017. [En línea]. Enlace: <http://bancroft.berkeley.edu/philobiblon/beta_es.html> [Consulta: 23/03/2018]).

${ }^{3}$ Su descripción en Julián Zarco Cuevas, Catálogo de los manuscritos castellanos de la Real Biblioteca de El Escorial, I, Madrid, Imprenta Helénica, 1924, pp. 202-206, y en Philobiblon BETA, manid 1548 (Ch. B. Faulhaber, Á. Gómez Moreno et al., ob. cit. [Consulta: 23/03/2018]). Acerca de la pertenencia del códice a los fondos reales, vid. Elisa Ruiz García, Los libros de Isabel la Católica: arqueología de un patrimonio escrito, Salamanca, Instituto de Historia del Libro y de la Lectura, 2004, pp. 386 y 498 [D2 42].
} 
Interesante es también la asociación codicológica con el entorno literario del Marqués de Santillana, promotor de la Qüestión a partir de sus lecturas del De militia de Leonardo Bruni; así se aprecia en el recuperado ms. Add. 9305 de la Cambridge University Library, donde al intercambio epistolar sobre la caballería le anteceden los Proverbios del Marqués, con su glosa y la de Pero Díaz de Toledo ${ }^{4}$. Podemos mencionar también el ms. BNE 27, que en su configuración original reunía, junto a Los doce trabajos de Hércules de Enrique de Villena, el Doctrinal y los Proverbios 5 .

En cuanto al Doctrinal, no es infrecuente que por su extensión o por interés de sus más conspicuos lectores, los miembros de la nobleza castellana, se haya difundido en códices de carácter unitario, con un solo texto de un único autor $^{6}$. La paradoja reside en que, siendo él mismo una compilación de textos legales atinentes al oficio de la caballería (desde las Siete Partidas y el Fuero Real de Alfonso el Sabio, hasta el Ordenamiento de Alcalá y la Orden de la Banda de Alfonso XI), su pluralidad conspira en ocasiones contra su correcta identificación o delimitación?

No obstante, entre las particularidades del Doctrinal conviene recordar que integra uno de los contados testimonios cuatrocentistas en lengua castellana (no facticios o copias tardías) en que Alfonso de Cartagena figura como autor único de más de un texto. Nos referimos a BNE $6609^{8}$, donde en

\footnotetext{
${ }^{4}$ Para localización, descripción y avatares de este manuscrito, olim Phillipps 8257, vid. Elena Carrillo Bermejo, «Descripción codicológica de un manuscrito recuperado con dos obras del marqués de Santillana: Ms. Add. 9305, Cambridge University Library», en Revista de Literatura Medieval, 23 (2011), pp. 311-331.

${ }^{5}$ Inventario General de Manuscritos de la Biblioteca Nacional, I (1-500), Madrid, Ministerio de Educación Nacional-Dirección General de Archivos y Bibliotecas, 1953, pp. 39-40, y Philobiblon BETA, manid 1624 y 1919 (Ch. B. Faulhaber, Á. Gómez Moreno et al., ob. cit. [Consulta: 24/03/2018]). Referencia completa de la sección desgajada de BNE 27 en Manuel Moreno, «Descripción codicológica ML4, CsXV I: 589. Ms. 660: Biblioteca Fundación Lázaro Galdiano, Madrid», como parte del proyecto dirigido por Dorothy Severin, An Electronic Corpus of the $15^{\text {th }}$ Century Castilian Cancionero Manuscripts. [En línea]. Enlace: <https://cancionerovirtual.liv.ac.uk/AnaAdditional/dutton/msdesc/ML4.pdf $>$ [Consulta: 24/03/2018]. Vid. et. M. ${ }^{a}$ Morrás, art. cit., p. 120, y María Morrás, «Fortuna de los Proverbios de Santillana: de la historia del texto a la historia de la recepción», en La escondida senda. Estudios en homenaje a Alberto Blecua, Barcelona, Castalia-Edhasa, 2012, pp. 39-62.

${ }^{6}$ Philobiblon BETA, texid 1438 (Ch. B. Faulhaber, Á. Gómez Moreno et al., ob. cit. [Consulta: 24/03/2018]), y Noel Fallows, The Chivalric Vision of Alfonso de Cartagena: Study and Edition of the Doctrinal de los caualleros, Newark, Juan de la Cuesta, 1995, pp. 47-69.

${ }^{7}$ Ejemplo de ello en la entrada del ms. RAH 9-712 en Antonio Rodríguez Villa, Catálogo general de manuscritos de la Real Academia de la Historia (1910-1912), versión digital 2005 de Juan Manuel Abascal, pp. 78-79. [En línea]. Enlace: $<$ http://www.rah.es/wpcontent/uploads/2016/09/Cat_gen manuscritos1.pdf> [Consulta 25/03/2018]. Descripción completa del Doctrinal en Philobiblon BETA, manid 1660 (Ch. B. Faulhaber, Á. Gómez Moreno et al., ob. cit. [Consulta: 24/03/2018]).

${ }^{8}$ En Inventario General de Manuscritos de la Biblioteca Nacional, XI (5700-7000), Madrid, Ministerio de Cultura-Dirección General del Libro y Bibliotecas, 1987, pp. 223-224, y en Philobiblon BETA, manid 3277 (Ch. B. Faulhaber, Á. Gómez Moreno et al., ob. cit. [Consulta: 23/03/2018]). Para una relación
} 
consideración de Noel Fallows el primero, la Qüestión, podría funcionar «as a seemingly pertinent introduction» respecto del segundo, el Doctrinal ${ }^{9}$.

Fallows también advierte en el Doctrinal la continuidad de algunos conceptos, como los deberes del caballero en la defensa de la fe, el combate contra el infiel y la extensión de las fronteras de la Cristiandad, expuestos previamente por Cartagena en el Concilio de Basilea (1434) en su discurso sobre la prceminentia sedium, esto es, en la Proposición contra los ingleses ${ }^{10}$.

Mas deseamos observar aquí que esta relación entre los tres textos que bien advierte Fallows ha quedado plasmada en una unidad codicológica escasamente conocida fuera de los catálogos de la institución que la conserva ${ }^{11}$. Nos referimos al ms. 389 de la Biblioteca Casanatense de Roma ${ }^{12}$, del que circa 1793-1794 daba noticia el jesuita español Lorenzo Hervás y Panduro' ${ }^{13}$.

de los testimonios de la Qüestión véase también Ángel Gómez Moreno, «La Qüestión del Marqués de Santillana a Don Alfonso de Cartagena», en El Crotalón, 2 (1985), pp. 335-363, esp. 340-342.

${ }^{9}$ Noel Fallows, «Chivalric manuals in medieval Spain: the Doctrinal de los caualleros (c. 1444) of Alfonso de Cartagena», en Journal of Medieval and Renaissance Studies, 24 (1994), pp. 52-87; cita en p. 62.

${ }^{10}$ N. Fallows, ibídem, pp. 62, 69 y 74.

${ }^{11}$ De hecho, no figura ni en Philobiblon ni en los repertorios bibliográficos dedicados a Cartagena, cfr. María Morrás, «Repertorio de obras, mss. y documentos de Alfonso de Cartagena (ca. 1384-1456)», en Boletín Bibliográfico de la Asociación Hispánica de Literatura Medieval, 5 (1991), pp. 213-248, y M. Morrás, art. cit., pp. 114-122. Tampoco lo mencionan o utilizan los editores de los tres textos involucrados: Alonso de Cartagena, Doctrinal de los caballeros, ed. de José María Viña Liste, Santiago de Compostela, Universidad de Santiago de Compostela, 1995; N. Fallows, ob. cit.; Alonso de Cartagena, Tratados Militares, ed. de Noel Fallows, Madrid, Ministerio de Defensa, 2006; Á. Gómez Moreno, ob. cit.; Marqués de Santillana, Obras completas, ed. de Ángel Gómez Moreno y Maxim. P. A. M. Kerkhof, Barcelona, Planeta, 1988, pp. 414-434; María Victoria Echevarría Gaztelumendi, Edición crítica del discurso de Alfonso de Cartagena «Propositio super altercatione prceminentia sedium inter oratores regum Castella et Anglice in Concilio Basiliense»: versiones en latín y castellano, Tesis doctoral, Madrid, Editorial de la Universidad Complutense, 1992. No se encuentra noticia en María Teresa Cacho, «Manuscritos hispánicos de los siglos XV y XVI en bibliotecas italianas», en Nápoles-Roma 1504. Cultura y literatura española y portuguesa en Italia en el Quinto Centenario de la muerte de Isabel la Católica, ed. de Javier Gómez-Montero y Folke Gernert, Salamanca, el SEMYR-la SEMYR-CERES de la Universidad de Kiel, 2005, pp. 189-203, ni en otras de sus publicaciones, dedicadas a diferentes fondos y bibliotecas de Italia. Sin dudas, el mayor interés de la crítica por los manuscritos hispánicos de la Casanatense ha estado centrado en el Cancionero de Roma, ms. 1098 (Dutton RC1) y el Cancionero Musical de la Casanatense, ms. 5437.

${ }^{12}$ Sobre la librería fundada por el cardenal Girolamo Casanate (1620-1700) y entregada en donación a los padres dominicos del Convento de S. Maria sopra Minerva en Roma, nos limitamos a consignar en este breve espacio los estudios de Pio Tommaso Masetti, «La Biblioteca Casanatense», en Memorie Domenicane, 48 (1931), pp. 280-289, 50 (1933), pp. 347-362 y 51 (1934), pp. 235-250, y Vincenzo De Gregorio, La Biblioteca Casanatense de Roma, Napoli, Edizioni scientifiche italiane, 1993. El ms. 389 no solo se encuentra en los registros antiguos, sino también en el catálogo electrónico. [En línea]. Enlace: $<$ http://opac.casanatense.it/Record.htm?idlist $=\&$ record $=10178421124929966039>$ [Primera consulta: 1/02/2015; última consulta: 6/04/2018]. La ficha online ofrece un enlace a la más reciente y extensa descripción de Maddalena Ceresi, Catalogo dei manoscritti della Biblioteca Casanatense, IV, Roma, Istituto Poligrafico dello Stato, 1961, p. 112.

${ }^{13}$ Lorenzo Hervás y Panduro, Catálogo de manuscritos de escritores españoles y portugueses existentes en siete bibliotecas insignes de Roma (III. Casanatense), Ms. BNE 13459, ff. 26r-55r; las citas en ff. $26 \mathrm{v}$ y $51 \mathrm{r}$. 


\section{Georgina OLIVETTO}

Alphonsus episcopus Burgensis, qui floruit in tempore concilii Basilensis. Proposicion al concilio de Basilea sobre la preeminencia del rei de España sobre el rey de Inglaterra. Respuesta á la qüestion hecha por el Marques de Santillana sobre el doctrinal del Cauallero. Codex chartaceus pulcher saeculi XIV. Miscellanea in folio: num. 40.

Santillana Marqués de. Carta al obispo de Burgos con una question sobre el doctrinal del Cauallero. Sub initium. Miscellanea in folio. num. 40 .

Con esta misma disposición, que da cuenta de solo dos textos al unificar la Qüestión y el Doctrinal, también lo encontramos en la que muy probablemente fuera la referencia de Hervás y Panduro, el primer catálogo sistemático del fondo manuscrito compilado en 1744 durante la prefectura de Gian Domenico Agnani ${ }^{14}$ :

Alphonsus (Alfonso don) Obispo de Burgos. vivens tempore Conc. Basileensis.

Proposition al Concilio de Basilea sobre la preheminencia del Rey d'Espagna sobre el Rey d'Ingleterra. Exst. in Cod. Chart. characteris nitidi illius seculi .XIV. Misc. in fol ${ }^{\circ} . \mathrm{n}^{\circ}$. 40. E.IV.38.

Respuesta a la question fecha por el Marques de Santillana sobre el Dottrinal del Cauallero. Exst. in eodem Codice.

La distinción de los tres títulos, aunque con un raro aserto sobre la autoría del Doctrinal, podemos localizarla tras más de un siglo en el catálogo dispuesto por Pio Tommaso Masetti, prefecto de 1872 a $1884^{15}$ :

E.IV.38. Miscellanea di Opuscoli in lingua Spagnuola. *1 El doctrinal de los Cavalleros.

\footnotetext{
${ }^{14}$ Index Codicum Manuscriptorum et Materierum eorundem (Antico inventario dei Manoscritti, Inventario 50), f. 9r. Idem en el volumen que integra este núcleo central con sucesivas actualizaciones, el Index Mss Bibliothecae Casanat. a P. M. Agnani Praefecto Magna Paleographiae arte quoad vetustiores Codices Concinnatus. Deinde a P. Lectore Brini aliisque prosecutus, et Anno 1844 a Rmo. P. M ${ }^{o}$. Hyacinto de Ferrari Bibl. Praef. absolutus, Tomo A-B, f. 21r (olim 41r). Una compendiosa historia de estos catálogos en Paola Busonero, Elisabetta Caldelli et al., I manoscritti datati delle biblioteche Casanatense e Vallicelliana di Roma, Firenze, SISMEL-Edizioni del Galluzzo, 2016, pp. 8-14.

${ }^{15}$ Codices Bibliotheca Casanatensis Bibliographice, et Paleographice illustrati per P. F. Pium Thom. Masetti O.P. ejusdem Praefectum Ab anno 1872 Ad annum 1878. Copia del inventario manuscrito (ms. $5415)$ consultada por gentileza de los responsables de la Biblioteca Casanatense. De acuerdo con P. Busonero, E. Caldelli et al., ob. cit., p. 13: «Fu il Masetti, tra il 1872 e il 1878, a compilare l'ultimo catalogo di manoscritti, purtroppo parziale, relativo a quei volumi collocati negli "armari della prima camera", con l'intento di redigere un "indice ragionato dei manoscritti antichi", encomiabile sforzo, ma non privo di inesattezze».
} 
Codice cart ${ }^{\circ}$ dal sec. Xv. Contiene* 2 Carta de Marques de Santillan al Obispo de Burgos sovra el doctrinal de los Cavalleros- 3. Alfonso Obispo de Burgos proposition al Concilio de Basilea sobra la preheminentia del Rey de Yspagna sobra al Rey d'Angleterra. Infine del Dottrinale= Laus tibi sit Christe, quoniam explicit liber iste. Antonius de Ferrera. E' questi senza dubbio l'autore del dottrinale.

Una vez identificado el Doctrinal en este repertorio del siglo XIX, aunque con la Qüestión aún subordinada a él, no se observarán demasiadas variantes en los registros sucesivos hasta llegar al catálogo electrónico ${ }^{16}$. No puede hablarse, por tanto, de descubrimiento bibliográfico, sino de recuperación o recordatorio de la labor de tantos bibliotecarios que procuraron dejar asentada la presencia de Alfonso de Cartagena en esta rica biblioteca romana.

En los últimos años no han sido pocos los esfuerzos por sacar a la luz otros testimonios del Doctrinal, desde el ms. Gaml. Kongl. Saml. 2219 de la Real Biblioteca de Copenhague, con la versión reducida del texto ${ }^{17}$, hasta el membranáceo 444 I e 7 del fondo agustiniano del Archivo Nacional de Praga ${ }^{18}$, y el más reciente ejemplo del Ms. Add. 8586 de la Cambridge University Library ${ }^{19}$.

Lo que llama la atención en el caso del manuscrito casanatense es la referencia de Masetti al firmante «Antonius de Ferrera», aunque podría explicarse por un detalle que el mismo bibliotecario consigna: la rúbrica no aparece al final del volumen sino en el cierre del Doctrinal (f. 306bisr), en verdad el segundo de los tres textos del códice. Si consideramos que no se trata de un facticio, que la copia pertenece a una sola mano del siglo XV y que su presentación mantiene un criterio uniforme (tipo de papel, márgenes, grafías, reclamos, etc.), debemos extender el crédito de Ferrera al total, aunque por supuesto en calidad de copista y no de autor. Por otra parte, no es el único

${ }^{16}$ Como apuntamos, la ficha se encuentra en el catálogo online de la Casanatense, pero todavía no en el repositorio del fondo manuscrito de Italia, Manus OnLine, dependiente de Istituto Centrale per il Catalogo Unico delle Biblioteche Italiane e per le informazioni bibliografiche: $<\mathrm{https}$ ://manus.iccu.sbn.it/> [Consulta: 12/04/2018]. Puede advertirse que la Qüestión, aunque discriminada por incipit, permanece a la sombra del Doctrinal en los inventarios modernos.

${ }^{17}$ Noel Fallows, «El Doctrinal de los caualleros de Alfonso de Cartagena, según el Ms. Gaml. Kongl. Saml. 2219 de la Real Biblioteca de Copenhague», en Hispania, 54:3, n. ${ }^{\circ} 188$ (1994), pp. 1107-1135.

${ }^{18}$ Robert Archer, «Un códice desconocido del Doctrinal de los cavalleros de Alfonso de Cartagena», en Tirant, 14 (2011), pp. 59-71.

${ }^{19}$ James Michael Ellis, Chivalry and Crisis at the Court of Juan II of Castile: The Chivalric Writing of Alonso de Cartagena and his Contemporaries, Tesis doctoral, Cambridge, University of Cambridge, 2016, pp. 197-209. [En línea]. Enlace: <https://www.repository.cam.ac.uk/handle/1810/273349> [Consulta: 08/04/2018]. Ms. olim Phillipps 4302 y Frederick Thomas Norton, vid. Philobiblon BETA, manid 4933 (Ch. B. Faulhaber, Á. Gómez Moreno et al., ob. cit. [Consulta: 12/04/2018]). 
manuscrito que lleva firma similar, pues el también cuatrocentista Cancionero de Duque de Gor, Biblioteca Bartolomé March 23-7-1 (B89-V1-13), concluye en el f. $152 \mathrm{r}^{20}$ :

Deo graçias. Aqui se acaba el libro versificado que fizo e copilo el noble e virtuoso cauallero ferrnand perez de guzman e escriujolo anton de ferrera criado del señor conde dalua por mandado del muy magnjfico señor don frey ferrnand gomez de guzman comendador mayor dela orden de calatraua. E acabose de escreujr primero dia de março año del señor de mjll e quatroçientos e çinquenta e dos años.

Podría tratarse, desde luego, de una mera coincidencia onomástica, pero un cotejo caligráfico de los manuscritos de la Casanatense y de la Biblioteca Bartolomé March ofrece pocas dudas acerca de su mano responsable ${ }^{21}$. Y si bien no se poseen demasiados datos acerca de la identidad de Antonio de Ferrera, más allá de los que él mismo proporciona en el colofón, esto es, que fue criado del conde de Alba, Fernán Álvarez de Toledo, y que cumplió un encargo del comendador mayor de la Orden de Calatrava, Fernán Gómez de Guzmán, son bien conocidos los lazos de ambos personajes con el Marqués de Santillana ${ }^{22}$ y

\footnotetext{
${ }^{20}$ Agradecemos a Fausto Roldán, director de la Biblioteca Bartolomé March, el inmediato acceso a las copias de este manuscrito. Para el Cancionero (Dutton MM2) remitimos a Maria Mercè López Casas, «El Cancionero del duque de Gor», Medioevo y Literatura. Actas del V Congreso de la Asociación Hispánica de Literatura Medieval, ed. de Juan Paredes, Granada, Universidad de Granada, 1995, III, pp. 37-60; Maria Mercè López Casas, «Fernán Pérez de Guzmán», en Diccionario Filológico de Literatura Medieval Española. Textos y transmisión, ed. de Carlos Alvar y José Manuel Lucía Megías, Madrid, Castalia, 2002, pp. 498-517; María Jesús Díez Garretas, «Descripción codicológica de MM2. Cancionero del Duque de Gor, Biblioteca de la Fundación Bartolomé March Servera, ms. B89-IV-13», en CIM. Cancioneros impresos y manuscritos, Descripciones codicológicas, 2013. [En línea]. Enlace: $<\mathrm{http} / / \mathrm{www}$. cancioneros.org/adjuntos/ MM2.pdf> [Consulta: 03/05/2017], y Philobiblon BETA, manid 2064 (Ch. B. Faulhaber, Á. Gómez Moreno et al., ob. cit. [Consulta: 23/03/2018]). Para la edición de los textos de Fernán Pérez de Guzmán presentes en este Cancionero y las referencias al mismo, vid. María Jesús Díez Garretas y María Wenceslada de Diego Lobejón, Un cancionero para Alvar García de Santamaría. Diversas virtudes y vicios de Fernán Pérez de Guzmán, Tordesillas, Universidad de Valladolid-Instituto de Estudios de Iberoamérica y Portugal, 2000, y María Jesús Díez Garretas, «La Confesión rimada de Fernán Pérez de Guzmán: estudio y edición», en Revista de Cancioneros impresos y manuscritos, 3 (2014), pp. 1-131.

${ }^{21}$ El Cancionero se encuentra incompleto y con los folios desordenados, por lo que remitimos a López Casas, art. cit., 1995, y Díez Garretas, art. cit., 2013, para el orden de los textos allí contenidos. Por ser copia de una sola mano no se presentan dificultades para el examen caligráfico, donde se aprecian como particularmente distintivas y concurrentes las mayúsculas $A, E, P, T$ y las minúsculas $d, h, y$ y $z$, con igual terminación e inclinación de los astiles ascendentes y descendentes, así como la tilde diacrítica de trazo fino sobre la $i$ (vid. Apéndice).

22 Íñigo López de Mendoza dedicará a su primo y compañero de infancia, el conde de Alba, el Bías contra Fortuna, al tiempo que el tercer señor de Batres, Fernán Pérez de Guzmán, autor de los textos contenidos en el Cancionero, también se encontrará emparentado con los Mendoza a través de los Ayala, siendo el comanditario de esta copia del Cancionero uno de sus sobrinos, el maestre Fernán Gómez de Guzmán, a quien están dedicados los Loores de los claros varones de España que en él se conservan.
} 
la referencia a los «Ferreras» en el «Prohemio del Marqués al Conde d'Alva» en Bías contra Fortuna ${ }^{23}$ :

Quando yo demando a los Ferreras, tus criados e míos, e aun a muchos otros, Señor e más que hermano mío, de tu salud e de quál es agora tu vida $[\ldots]$ Con estos Ferreras me escreviste que alguno de mis tractados te enbiasse por consolación tuya.

La relación con la familia Santa María es igualmente notoria, por lo que solo recordaremos aquí que el Cancionero del duque de Gor constituye un corpus de composiciones de tema religioso y didáctico-moral destinado a Álvar García de Santa María, donde se incluyen las Diversas virtudes y vicios a él dedicadas ${ }^{24}$. Del mismo modo que algunas «questiones» formuladas por Fernán Pérez a su mentor intelectual y espiritual, Alfonso de Cartagena, son el punto de partida para la composición tanto del Oracional como del inconcluso Duodenarium, a él destinados, sin contar el común interés por Séneca y la indudable influencia de Cartagena como tratadista y traductor en la obra personal de Fernán Pérez ${ }^{25}$, quien así lo despedía, como Lucilio a su maestro,

Véanse al respecto M. ${ }^{a}$ M. López Casas, art. cit., 1995, pp. 40-45, y art. cit., 2002, pp. 498-501 y 508 (I MM2), así como M. J. Díez Garretas y M. ${ }^{a}$ W. de Diego Lobejón, ob cit., pp. 11-30. Añádanse otras dos contribuciones de Maria Mercè López Casas, «De la transmisión manuscrita de los Loores de los claros varones de España de Fernán Pérez de Guzmán», en Actas do IV Congresso da Associação Hispânica de Literatura Medieval (Lisboa, 1-5 Outubro 1991), ed. de Aires A. Nasçimento y Cristina Almeida Ribeiro, Lisboa, Cosmos, 1993, IV, pp. 169-174, y «Fernán Gómez de Guzmán: el envés histórico de la figura del Comendador de Fuenteovejuna», en Las órdenes militares: realidad e imaginario, ed. de María Dolores Burdeus, Elena Real y Joan Verdegal, Castellón, Universitat Jaume I, 2000, pp. 469-480.

${ }^{23}$ Ya lo señalaba M. ${ }^{\text {a }}$ M. López Casas, art. cit., 1995, pp. 40-41. La cita del texto en Marqués de Santillana, ed. cit., pp. 270-271.

${ }^{24}$ Así lo consigna la última copla de MM2 bajo la rúbrica «Ultilogo», f. 152r: «Dela gruesa jnuençion $\mathrm{mja}$ / e sinple ymaginaçion / ved aquj la rrelaçion / muy buen honbre aluar garçia / plega ala virgen maria / que sant iohan e sant benjto / al gozo dulçe jnfinjto / nos lieuen con alegria». De acuerdo con M. ${ }^{\mathrm{a}}$ J. Díez Garretas y M. ${ }^{a}$ W. de Diego Lobejón, ob. cit., pp. 48-49 y 54, esta obra comprendería el Prólogo, el cuerpo central de las coplas de virtudes y viçios, el poema mariano Oraçión a Nuestra Señora en fin de todo y el mencionado Ultílogo. La Oraçión habría sido escrita a fin de cerrar el conjunto del cancionero para Álvar García, aunque en MM2 solo se conserve su último verso.

${ }^{25}$ Información al respecto en las ediciones de Silvia González-Quevedo Alonso, El «Oracional» de Alonso de Cartagena: edición crítica (comparación del manuscrito 160 de Santander y el incunable de Murcia), Chapel Hill-Valencia, Albatros, 1983, y Luis Fernández Gallardo y Teresa Jiménez Calvente, El Duodenarium (c. 1442) de Alfonso de Cartagena. Cultura castellana y letras latinas en un proyecto inconcluso, Córdoba, Real Círculo de la Amistad-Almuzara, 2015, esp. «II. El porqué del Duodenarium», pp. 47-68. Para el perfil literario de Pérez de Guzmán y la influencia de Alfonso de Cartagena, vid. Francisco López Estrada, «La retórica en las Generaciones y semblanzas de Fernán Pérez de Guzmán», en Revista de Filología Española, 30 (1946), pp. 310-352, y Luis Fernández Gallardo, «Disidencia política y nuevos valores literarios en Generaciones y semblanzas», en En la España Medieval, 25 (2002), pp. 267297. Para el caso puntual de Diversas virtudes y vicios, M. ${ }^{a}$ J. Díez Garretas y M. ${ }^{a}$ W. de Diego Lobejón, ob cit., pp. 36-41. 
en las Coplas sobre el tránsito del reverendo padre don Alfonso de Cartage$n a^{26}$. La biblioteca del señor de Batres es también buen ejemplo de la fluida relación intelectual con los Santa María ${ }^{27}$.

En este marco presentamos entonces la descripción analítica del testimonio cuatrocentista rubricado por Ferrera, que seguramente será de interés para los especialistas.

\section{Roma, Biblioteca Casanatense, ms. 389 (olim E.IV.38)}

1. Íñigo López de Mendoza, Marqués de Santillana \& Alfonso de Cartagena, Qüestión sobre el fecho de la cavallería

[h. 1r, rub.]: Carta del Marques de | Santillana para el Re|uerendo señor don Alon|so Obispo de Burgos.

[h. 1r]: Leyendo yo este otro dia 9 Reuerendo señor $\$ e mi | mucho especial e grande amigo $\llbracket$ vna pequeña obra $\llbracket$ de $\mid$ Leonardo de Arezio $\llbracket$ en la qual ha querido mostrar

[h. 1v]: \ para deffension de su patria $\llbracket$ conuiene saber delos [recl.: estados]

f. 2r: estados / conujene saber labradores / $\tau$ ofiçiales $\llbracket$ Como | los otros / que dexados estos ofiçios sola mente quedaron por caualleros.

f. $3 \mathrm{v}$ : $\mid \mathrm{E}$ avos señor $\mid$ mjo faga enlos presentes $\tau$ aduenjderos tienpos a|legre / $\tau$ bien auenturado. a. xv. de enero año de .xl $\mid$.iiij ${ }^{\circ}$.

f. 4 [blanco]

f. $5 \mathrm{r}$ [rúbr.]: Respuesta del venerable $\tau$ sabio señor don alfon|so obispo de burgos / ala quistion / fecha por el magnj|fico señor / marques de santillana.

f. 5r: Con quanta alegria / o muy claro varon ley | vuestra graçiosa letra / de aquello se puede con $\mid$ siderar que muchas causas enella concurrieron

\footnotetext{
${ }^{26}$ Nótese que esta composición también se transmite en códices con marcada impronta de los Santa María, como el ya mencionado Esc. h-II-22 (Manid 1548), Esc. Y-III-8 (Manid 1672), donde comparte folios con el Oracional y la correspondiente Letra mensajera de Fernán Pérez, y BUS 2198 (Manid 1673) que, aunque facticio de los siglos XV-XVI, reúne la Proposición contra los ingleses y la Glosa al tractado de san Juan Crisóstomo. En similares compañías llegará a la imprenta, cfr. la edición del Oracional de Murcia, Gabriel Luis Ariño y Lope de la Roca, 1487 (Manid 1653). Para todo ello, Philobiblon BETA, texid 1702 (Ch. B. Faulhaber, Á. Gómez Moreno et al., ob. cit. [Consulta: 15/04/2018]).

${ }^{27}$ Fernán Pérez de Guzmán, Generaciones y semblanzas, ed. de Robert Brian Tate, London, Tamesis, 1965, pp. XI-XII; Gregorio de Andrés, «Los códices que vio Ambrosio de Morales en el Castillo de Batres en 1572», en Anales del Instituto de Estudios Madrileños, 33 (1993), pp. 267-276, y Mercedes Vaquero, «Cultura nobiliaria y biblioteca de Fernán Pérez de Guzmán», en Anexos de la Revista Lemir, 7 (2003). [En línea]. Enlace: <http://parnaseo.uv.es/Lemir/Revista/Revista7/Vaquero/MercedesVaquero.htm> [Consulta: 06-02-2018], vid. Apéndice I, ítems 7, 14, 23, 30, 53 y Apéndice II, ítems 12, 13, 18b, $26 \mathrm{a}, 27$.
} 
f. 19v: $\tau$ aqujen la eglesia adorando / canta / san|to santo santo / señor dios delas huestes / amen $\uparrow \mid$ de burgos A .xvij. dias de março de .xliiijo .

f. 20 [blanco]

\section{Alfonso de Cartagena, Doctrinal de los cavalleros}

f. 21r [Tabla]: - T Tabla delos titulos deste libro que se llama el doctrinal delos caualleros / el qual es partido en quatro libros.

f. $21 \mathrm{r}$ [rúbr.]: libro primero en que ay nueue titulos.

f. 21r: $\mid$ Titulo primero dela santa trinjdad $\tau$ dela fe catholica $\mid$ en que ay dos leyes.

f. 23r: \ Rubriçela sesta del traher delas vestiduras enque ay v|na ley.

f. $23 r$ [rúbr.]: Ay nueue titulos $\tau$ çiento $\tau$ dos leyes.

f. $23 \mathrm{v}$ [blanco]

f. $24 \mathrm{r}$ [rúbr.]: Aqui comjença vna copilaçion de aquellas leyes del rreg|no de castilla que fablan delos fechos que tañen alos $\mid$ caualleros $\tau$ fijos dalgo.

f. $24 \mathrm{r}$ [rúbr.]: libro primero

f. 24r: Los famosos caualleros muy noble señor condesta|ble que enlos tienpos antigos por diuersas rre|giones del mundo floresçieron

f. 28r: jnterponjendo algunas | vezes entre vnas $\tau$ otras / palabras suyas para mejor li|gar $\tau$ continuar la jnsertura $\llbracket$ dexemos ya esto $\tau$ comen|çemos lo que queremos fazer.

f. $28 \mathrm{r}$ [rúbr.]: Titulo primero dela san|ta trinjdad $\tau$ dela fe catholica en que ay dos leyes. Introduçion. / en que ay .ij. leyes.

f. $28 \mathrm{v}$ : Rayz de todos los bienes $\tau$ comjenço de todas las bu|enas obras $\tau$ firmeza de todas las leyes / es la $\mid$ santa fe catholica

f. 305v: $\tau$ que non trayan oro enlas vandas njn | enlos paños / njn en otra Cosa alguna.

f. $305 \mathrm{v}$ [rúbr.]: Conclusion.

f. 305v: Estas leyes / muy virtuoso cauallero copille | asi / por vuestro mandado / apartando desus origi|nales aquellas que ala caualleria / o a actos de cauallleros $\tau$ fijos dalgo paresçian fazer

f. 306bisr: ๆ Por ende como qujer que ello sea / | vos de buena mente açeptad este pequeño trabajo que $\mid$ por mandamjento vuestro con alegre coraçon $\tau$ presta ma|no / en esta conposiçion yo tome.

f. 306bisr [colofón] [rúbr.]: laus sit tibi xpe | quoniam explicit liber iste

f. 306 bisr [colofón]: Antonjus de ferrera

f. 306bisr [blanco] 


\section{Alfonso de Cartagena, Proposición contra los ingleses}

f. 307r: ๆ Este es traslado de vna muy solepne proposiçion que | el señor don alfonso obispo de burgos / fizo contra los jngle|ses seyendo ambaxador enel conçilio de basilea I sobre | la prehemjnençia que el rrey nuestro señor ha sobre el rrey | de jnglaterra / la qual arruego del señor iohan desilua al|ferez mayor del dicho señor rrey $\tau$ su conpañero enla | enbaxada / el torno de latin en rromançe.

f. 307r: Mienbraseme muy rreuerendos padres de aque|lla rrazon que dizen que dixo demostenes. | E avn que non rrepita las palabras / dire el efecto | segun que ala memoria me ocurre.

f. 340v: E asi paresçe que nuestra jntençion queda en su firmeza non enbargante lo en $\mid$ contrario allegado.

f. 340v: I Esto es muy rreuerendos padres lo que en esta mate|ria me ocurre de presente 9 Pero si alguno ay que en | contrario qujera otras cosas allegar aparejado so arres|ponder non esforçandome enlas fuerças de mj jngenjo el | qual çierta mente es rrudo $\tau$ obscuro / mas confiando / en | la ayuda djujnal $\tau$ enla fortaleza dela verdad.

Siglo Xv. Papel; filigranas: peregrino o caminante inscrito en círculo, con letras BL al pie [h. 1 añadida]; en el resto del códice, variantes de flor «en forme de tulipe ou de campanule» con dos hojas [ej. ff. 3, 20, 187; Briquet II, pág. 376: «marque exclusivement italienne»]; 267 x 205-210 mm., caja pautada con lápiz de plomo y renglones a punta seca, 185 x $120 \mathrm{~mm}$. [f. 156], 1 columna, 25 líneas. Códice muy homogéneo en formato y escritura ${ }^{28}$.

$1 \mathrm{~h}+1^{4-1}, 2^{12-1}, 3^{6-1}, 4^{12}, 5^{12}, 6^{12}, 7^{12}, 8^{12-1}, 9^{12}, 10^{12}, 11^{12}, 12^{12}, 13^{12}, 14^{12}, 15^{12}$, $16^{12}, 17^{12}, 18^{12}, 19^{12}, 20^{12}, 21^{12}, 22^{12}, 23^{12}, 24^{12}, 25^{12}, 26^{12}, 27^{12}, 28^{12}, 29^{12}, 30^{10-2}$ $=341 \mathrm{ff}$.

Reclamos horizontales en todos los cuadernillos, excepto en los que finalizan texto con folio blanco: 1 blanco (fin de la Carta de Santillana), 2 'non' (f. 15v), 3 blanco (fin de la Respuesta de Cartagena y de la Qüestión), 4 'de' (f. 32v), 5 'que' (f. 44v), 6 'se' (f. 56v), 7 'ria' (68v), 8 'turas' (79v), 9 ' $\tau$ ' (f. 91v), 10 'en' (f. 103v), 11 'en' (f. 115v), 12 'A' (f. 127v), 13 'la' (f. 139v), 14 'de' (f. 151v), 15 'por' (f. 163v), 16 'se' (f. 175v), 17 'el' (f. 187v), 18 'o' (f. 199v), 19 'lo' (f. 211v), 20 'lante' (f. 223v), 21 'non’ (235v), 22 'que’ (f.

\footnotetext{
${ }^{28}$ Vid. la descripción sumaria del códice en M. Ceresi, ob. cit., p. 112. Aunque se indica el orden, no se lo guarda en la descripción de los contenidos, que comienza por el Doctrinal.
} 
247v), 23 'lla' (f. 259v), 24 'sy' (271v), 25 'tria' (f. 283v), 26 'ler' (f. 295v), 27 blanco (f. 306bisv, fin del Doctrinal), 28 'creto' (f. 318v), 29 'E' (f. 330v), 30 (fin de la Proposición).

Los cuads. 1-3, que contienen la Qüestión, forman una primera unidad. Falta el f. 1, reemplazado por una hoja de papel verjurado más fino, sin numeración visible, con texto repuesto por mano humanística, con uso de tinta negra ferrogálica que ha dañado considerablemente el papel, y con título, inicial y calderones en tinta roja. Entre ff. 16 y 17, se aprecia la pestaña de la hoja añadida y restos de un folio cortado. Los cuads. 1-2 han sido recosidos para la inclusión de la mencionada hoja inicial, por lo que no se aprecia la estructura primigenia; no hay más pérdida de texto, de modo que los demás folios faltantes debieron estar en blanco.

En el cuad. 8 falta el folio décimo del senión (entre actuales ff. 77-78), con pérdida de texto. La numeración moderna no lo advierte, pero sí se aprecia el salto en la numeración antigua del lxxvij al lxxjx. Un trozo de papel insertado modernamente, con tinta negra, da cuenta de la pérdida: «manca una c. tra la 77 e la 78», al tiempo que apunta el salto entre «c. 307-308».

En el cuad. 30 y último faltan 2 folios, sin pérdida de texto, probablemente los dos últimos blancos del senión.

Foliación antigua en romanos, tinta negra, ángulo inferior derecho del recto, refilada en los primeros folios. Foliación antigua en arábigos, tinta negra, centro del margen superior del verso, del 2 al 10. En los folios que comprenden el Doctrinal, indicación de capítulos en el margen superior (recto y verso), tinta negra, en general refilada. Foliación moderna en arábigos, con sello de tinta negra, ángulo superior derecho del recto, que no considera el $\mathrm{f}$. lxxviij perdido y salta un folio entre cccvij y cccviij, corregido posteriormente a lápiz como 306bis. Seguimos, no obstante, esta última foliación, para facilitar una rápida ubicación de los contenidos

La numeración antigua olvida numerar un folio entre los ff. 212 y 213, por lo que se empareja con la numeración moderna hasta el f. 306.

Letra gótica redonda del s. Xv, tinta negra. Iniciales, títulos, epígrafes y calderones en tinta roja. Iniciales simples en rojo con rasgueos de pluma en negro. Una mano contemporánea de la copia, con tinta más oscura, anota en los márgenes de la Tabla del Doctrinal las correspondencias de partes, títulos y capítulos, así como las referencias de las fuentes legales, a los que luego remite en los márgenes del texto; de la misma mano parecen ser algunas correcciones marginales al texto, que aludirían a un cotejo con otro testimonio de lecciones divergentes (f. 88r: «al. desapoderada» frente al texto «desperada»; 
f. 91r: «al. naucheles» frente al texto «Nocheros»; f. 138r: «al. leuasen» frente al texto «fuesen»; f. 173v: «al. tener» frente al texto «tomar»).

Encuadernación flexible en pergamino, correíllas de cuero faltantes.

Sobre la cubierta anterior, margen superior, con tinta negra y pluma fina: «400 | doctrinal de cavalleros»; en el ángulo inferior derecho: «35 1».

Lomo de cuatro nervios con refuerzos internos en pergamino; en la cabeza, con tinta negra, excepto la L mayúscula en rojo: «di | Leonardo d'| Arezio Dottri | nal del Caualle ${ }^{\mathrm{ro}} 》$; debajo, otra mano, con tinta más clara: «Marques

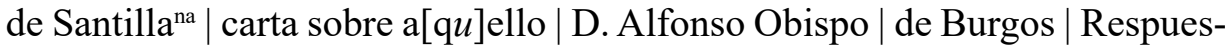
ta $\mid$ y $\mid$ Propos $^{\text {on }}$ al Concilio de | Basilea sobre la | Preheminencia del Rey d' Espanna al Rey d' | Inglaterra | MS.»; debajo, otra mano, tinta negra: «Miscell. in f. 40 | E. IV. 38» (s. XVIII); al pie, estampilla de la Regia Biblioteca Casanatense Roma, con la signatura «Mss. 389».

Cortes moteados; en el corte inferior, tinta negra: «Dotrinal de Cauallero | Spagnolo.».

Una guarda inicial y otra final, ambas blancas, con filigrana de corazón con cruz e iniciales AG inscritas. En la guarda pegada anterior, ángulo superior izquierdo, distintas manos consignan sucesivamente, en tinta negra, las signaturas: « $\mathrm{B}^{\mathrm{co}}$. S. 1 35» [cancell.], «E. IV. 38.», «Cod. 389»; en el centro, misma estampilla del lomo de la Biblioteca Casanatense con signatura actual. En la guarda volante anterior, recto, margen superior, con tinta negra: «C. 68», que se repite de igual modo en la h. 1r; en el vuelto, con tinta negra, relación de los contenidos del códice y, de otra mano, apuntes sobre la escritura (contemporánea del Concilio de Basilea, s. Xv) y sobre quien suscribe el Doctrinal (Antonius de Ferrera), atribuibles a los catalogadores del Index Mss. (todas notas añadidas en Italia) ${ }^{29}$.

Sello ovalado en tres tamaños (h. 1, ff. 23r, 250r y f. 307r), con las armas del cardenal Girolamo Casanate (s. XVII): torre almenada a la gibelina y estrella de ocho puntas superpuesta, con iniciales «H.C.C.» en torno.

Códice en buen estado de conservación. Actual f. 2 manchado y con los márgenes del papel más dañados, lo que indica una pronta pérdida del primer folio original. Escasas anotaciones marginales, excepto marcas de lectura paralelas a los párrafos, algunas de notatur (f. $48 \mathrm{v}$, también con breve nota marginal); anotaciones extensas en castellano de la misma mano cursiva del s. Xv en ff. $37 \mathrm{v}, 310 \mathrm{r}$ y $324 \mathrm{v}$, donde se aprecia el refilado del corte externo del

${ }^{29}$ M. Ceresi, ob. cit., p. 112: «Nel v. della $1^{\text {a }}$ c. di guardia, di mano del bibliotecario casanatense (forse il P. Agnani), il contenuto del codice e una nota sull'Autore, erroneamente ritenuto Leonardo d'Arezzo, di cui viene fatta menzione nella prima delle due lettere che precedono il testo». 
códice; otro apunte, de diferente mano cursiva, en f. 276r (vid. infra). Pruebas de pluma en el verso del último folio.

No se observan blancos producidos por lagunas y nótese que en el Doctrinal, en el pasaje que cita Archer acerca del uso del término «combatir», no solo no se deja el blanco sino que se ofrece una lección diferente a la del ms. 444 I e 7 del Archivo Nacional de Praga («Et el envarrar es dicho»), que coincide con la lectura de otros testimonios y con la que adopta Fallows ${ }^{30}$ :

mas antigua mente los de españa este nonbre mudaron en muchas maneras segun los fechos de armas eran e segun los ombres que los fazian e por ende el conbatjr que dixjemos toujeron que non conuenja para dezjr la sobre otra cosa sinon quando conbaten alguna fortaleza que qujeren tomar. E el conbatjr es dicho quando los enbarran de manera que por njnguna parte non osan saljr de gujsa que los han despues a entrar por fuerça (f. 82v).

Si bien en el ms. Casanatense el texto aparece dedicado al «muy noble señor condestable» don Álvaro de Luna, no hay modificaciones en la segunda referencia de la Introducción, como sí detecta Archer en el ms. de Praga («E como vos el dicho señor condestable de la primera manera») ${ }^{31}$, pues se mantiene simplemente el «vos»: «E como vos dela primera manera» (f. 26v). En cuanto a las propiedades de Castro y Lerma pertenecientes al primer dedicatario, Diego Gómez de Sandoval, tampoco hay cambios en la Introducción al Título IV: «segun la qual llamamos vasallos solariegos alos que bjuen en logar de algun señor que non es behetria como son vuestros los de castro e de lerma e de otros logares» (f. 276r) ${ }^{32}$. Destaca en nuestro manuscrito una nota en el margen externo que expresa esta misma incongruencia respecto de la dedicatoria precedente al Condestable (mano cursiva posterior, en tinta más clara; afectada por el refilado):

puesto que la yntroduçion [de]ste libro dize as[y?] del condestable notar que fue fe [cho] a ynstançia del muy virtuoso y [mu]y esforçado ca[ba]llero y señor el se[ñor] conde de castro y denja lo qual ma[ni]fiest $a$ aquello que d[ice] commo son los vuestr[os] de castro y de [ler]ma de muchas bi[llas] era señor con [o]tras diez billas [çer] cadas » ${ }^{33}$.

${ }^{30}$ Fallows, ob. cit., 1995, p. 139, a partir de Burgos, Fadrique de Basilea, 1487, f. E ${ }_{4}$. Archer, art. cit., pp. 70-71.

${ }^{31}$ Archer, art. cit., p. 70.

${ }^{32}$ Cfr. Fallows, ob. cit., 1995, p. 327 y n. 2.

${ }^{33}$ Vid. Ismael García Rámila, Estudio histórico-crítico sobre la vida y actuación politico-social del burgalés ilustre que se llamó D. Diego Gómez de Sandoval, Adelantado Mayor de Castilla y primer conde de Castro y Denia (1385-1455), Burgos, Imprenta de la Excma. Diputación, 1953, p. 75, respecto de la 
En cuanto a algunas variantes y lagunas significativas (aquí sin blancos visibles) que enmienda Fallows en su edición sobre el texto base del incunable de Burgos, 1487, damos cuenta, a manera de ejemplo, $\mathrm{de}^{34}$ :

Libro I, Título III (p. 100, n. 32): «E porende pues que los caualleros nobles tan grande debdo han conlos queles desçiñen las espadas [...] aqujen han de rrogar que sean sus padrinos para desçeñjrgelas» (f. 43r).

Libro I, Título IV (pp. 107-108, n. 4-5): «pero en algunas tierras es nonbre de señorio señalado asi como en alemaña e en la morea e en antiocha e en napol» (f. 51r).

Libro I, Título VII (pp. 123-125, n. 12): No se registra la laguna (ff. 66v-68v). En el impreso puede deberse, en efecto, a un error en el manejo de los folios del manuscrito base $o$, añadiríamos, a una pérdida temprana de los mismos. Libro I, Título IX (pp. 154-156, n. 9): No se registra la laguna, con los dos items completos (ff. 97r-99r).

Libro IV, Prólogo (p. 310, n. 5-6): «E el apostol sant paulo en enla [sic] epistola de tito alego contra los de creta vn dicho del poeta epjmedes» (f. 259r). Libro IV, Título III (p. 322, n. 7): «Ca asy es escripto enla ley que njngun ombre non puede ser vasallo de dos señores» (f. 270r).

Recibido: $18 / 04 / 2018$

Aceptado: 22/06/2018

inscripción sepulcral del primer conde de Castro en el convento de Domus Dei de Aguilera: «Está aquí también el cuerpo del Adelantado mayor de Castilla primer conde de Castro, Diego Gómez de Sandobal, llamado por excelencia EL GRAN SEÑOR, que lo fué de diez villas cercadas en Castilla y uno de los más balerosos, prudentes y cuerdos caballeros de su tiempo, a quien el rey D. Juan de Nabarra y D. Alonso de Aragón dieron la villa de Denia, que hoy posee su Cassa y sucessor della con título de Marqués».

${ }^{34}$ Remitimos a continuación a Fallows, ob. cit., 1995, solo por número de página y nota. 
Tres títulos de Alfonso de Cartagena en la Biblioteca Casanatense de Roma

\section{Apéndice fotográfico}

Casanatense
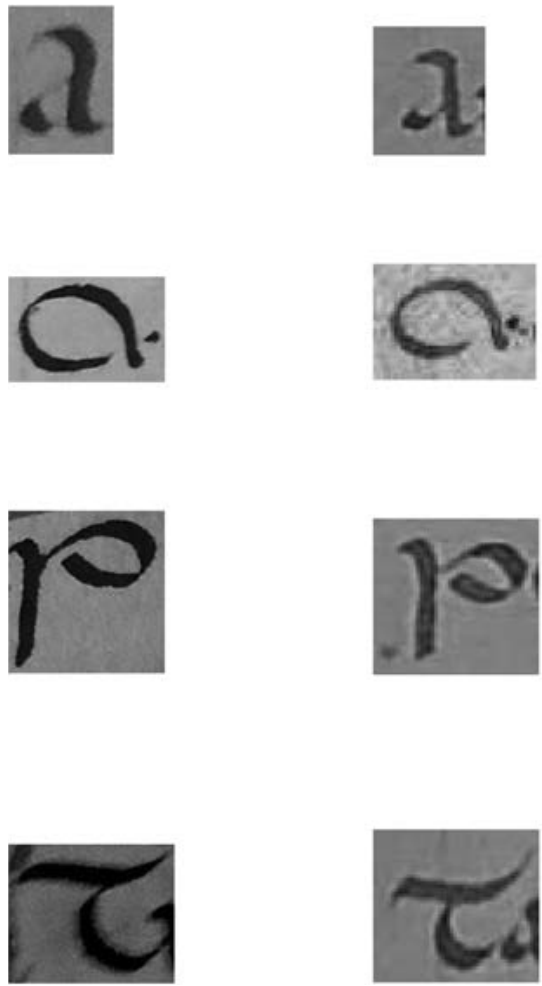

Casanatense

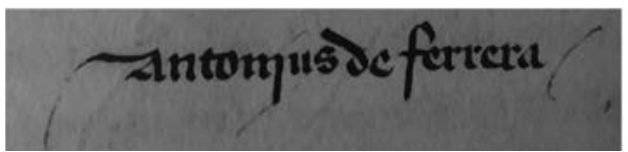

Casanatense

B. March
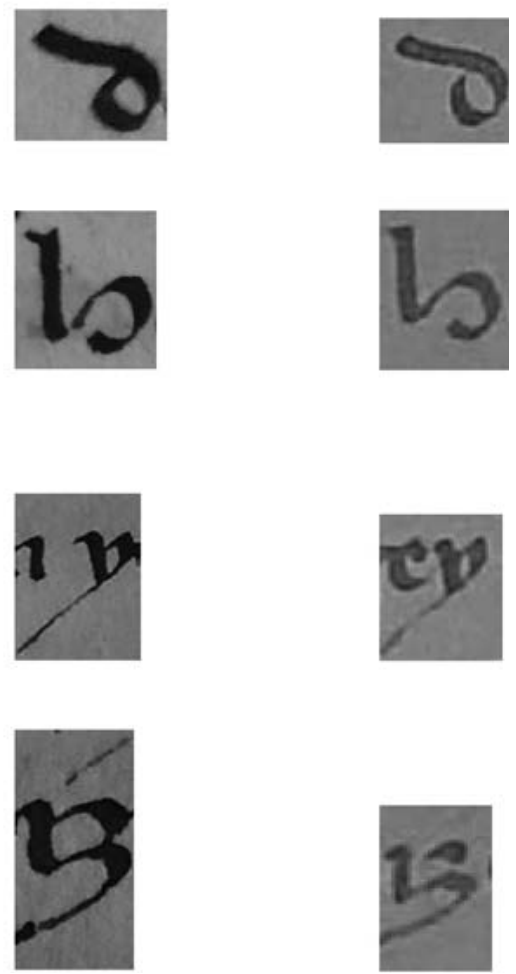

B. March

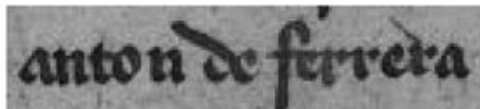




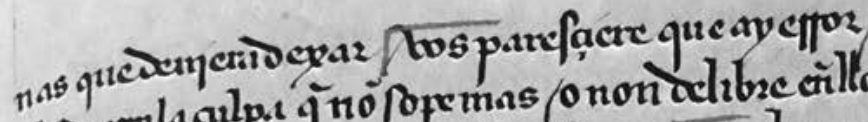

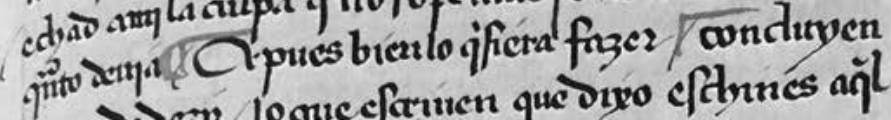

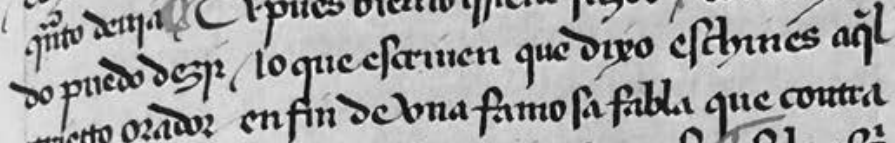

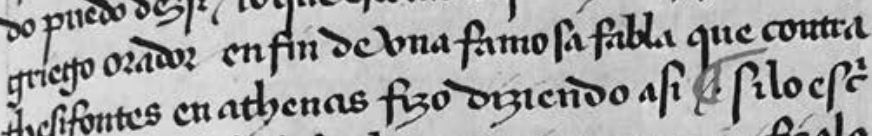

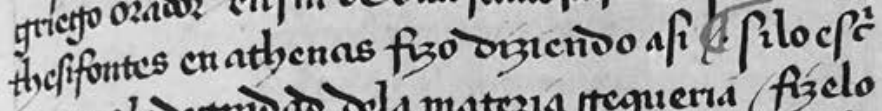
in comola Digmoro dela matezia fequeria fizclo pogut qupe 1 . finonlo eqauy tan bien fizelo

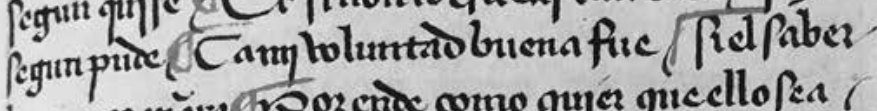

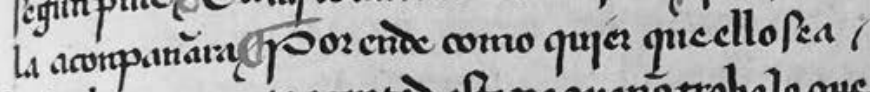
los debuena menteasaptrio efte pequenotraba] o que prmantramyento b2o con alegre wascon z preftama hopercefta corpoficion yo tome $(\div \quad \therefore \quad \therefore \quad \div$ $\because$

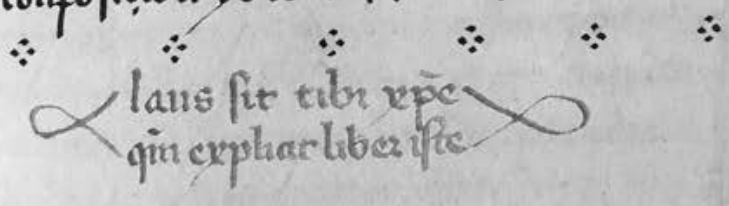

antomusdefertera

(C) Roma, Biblioteca Casanatense, ms. 389, f. 306bisr 
que ae buen functo ay a fifinenga
vltulogto

Dda gruefa purengon ma

= fmple nuagrmacion

veraquy la ffelacion

mu buenl joubre aluargaraa

plega alcr bryen mana

que fantolisu z ant herpto

al goso oulce jufinto

nos heuen con alegera

$$
\begin{aligned}
& \text { acdconde. } \\
& \text { torrepalma. }
\end{aligned}
$$

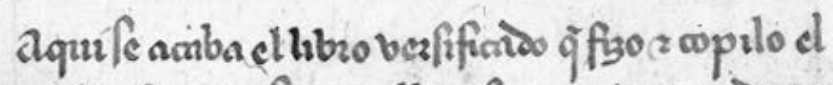

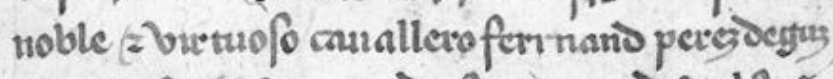
mar zefertuy olo anton de furvetra cmido del ferios

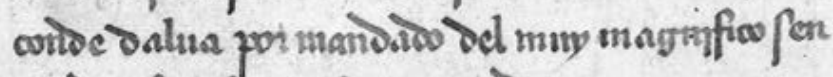

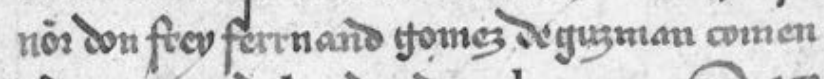
Datoor mayos dala oroen se callatraul. Oracu bofe se ef

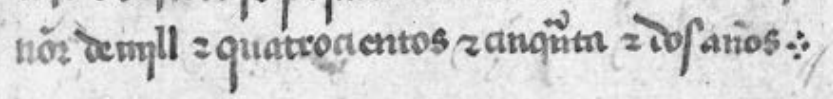

(C) Palma de Mallorca, Biblioteca Bartolomé March, ms. 23-7-1, f. 152r 


\section{$\cos$}

\section{Tres títulos de Alfonso de Cartagena \\ en la Biblioteca Casanatense de Roma (ms. 389)}

RESUMEN: Se ofrece información detallada del ms. 389 de la Biblioteca Casanatense, el cual reúne tres textos de Alfonso de Cartagena: Qüestión sobre el fecho de la cavallería, Doctrinal de los cavalleros y Proposición contra los ingleses. El interés de este manuscrito del siglo XV reside en que no figura en los repertorios bibliográficos (aunque sí en los catálogos de la Casanatense), no ha sido empleado por los respectivos editores de los textos y, fuera del muy compacto corpus de traducciones de Séneca y Cicerón, es uno de los pocos unitarios que conservan exclusivamente obras de nuestro autor. El ms. 389 presenta asimismo la particularidad de la firma «Antonius de Ferrera», que a partir de un cotejo caligráfico podría identificarse con el «Anton de Ferrera» que subscribe en 1452 el Cancionero del Duque de Gor.

Palabras clave: Manuscritos. Copistas. Alfonso de Cartagena. Biblioteca Casanatense.

\section{Three Titles of Alfonso de Cartagena in the Casanatense Library of Rome (MS. 389)}

ABSTRACT: The article offers detailed information about the ms. 389 of the Casanatense Library, which brings together three texts by Alfonso de Cartagena: Qüestión sobre el fecho de la cavallería, Doctrinal de los cavalleros and Proposición contra los ingleses. The interest of this Xvth century manuscript lies in the fact that it is not included in the bibliographic repertoires (although it does in the catalogues of the Casanatense), it has not been used by the respective editors of the texts and apart from the very compact corpus of translations of Seneca and Cicero it is one of the few unitaries that exclusively conserve works of our author. The ms. 389 also presents the particularity of the signature «Antonius de Ferrera», which from a calligraphic comparison could be identified with the «Anton de Ferrera» who subscribes in 1452 the Cancionero del Duque de Gor.

KeYwords: Manuscripts. Copyists. Alfonso de Cartagena. Casanatense Library. 